CUBO A Mathematical Journal Vol.12, $N^{\beta o} 03$, (213-239). October 2010

\title{
Strichartz Estimates for the Schrödinger Equation
}

\author{
Elena CORDERo AND DAVIDE ZuCCO \\ Department of Mathematics, University of Torino, \\ v. Carlo Alberto 10, Torino, Italy \\ email: elena.cordero@unito.it \\ email: davide.zucco@unito.it
}

\begin{abstract}
The objective of this paper is to report on recent progress on Strichartz estimates for the Schrödinger equation and to present the state-of-the-art. These estimates have been obtained in Lebesgue spaces, Sobolev spaces and, recently, in Wiener amalgam and modulation spaces. We present and compare the different technicalities. Then, we illustrate applications to well-posedness.
\end{abstract}

\section{RESUMEN}

El objetivo de este trabajo es reportar los progresos recientes sobre estimativas de Strichartz para la ecuación de Schrödinger y presentar el estado de arte. Estas estimativas han sido obtenidas en espacios de Lebesgue, espacios de Sobolev, y recientemente, en espacios de Wiener amalgamados y de modulación. Presentamos y comparamos los diferentes aspectos técnicos envueltos. Ilustramos los resultados con aplicaciones a buena colocación.

Key words and phrases: Dispersive estimates, Strichartz estimates, Wiener amalgam spaces, Modulation spaces, Schrödinger equation.

Math. Subj. Class.: 42B35,35B65, 35J10, 35B40. 


\section{Introduction}

In this note, we focus on the Cauchy problem for Schrödinger equations. To begin with, the Cauchy problem for the free Schrödinger equation reads as follows

$$
\left\{\begin{array}{l}
i \partial_{t} u+\Delta u=0 \\
u(0, x)=u_{0}(x)
\end{array}\right.
$$

with $t \in \mathbb{R}$ and $x \in \mathbb{R}^{d}, d \geq 1$. In terms of the Fourier transform, we can write the solution as follows

$$
u(t, x)=\left(e^{i t \Delta} u_{0}\right)(x):=\int_{\mathbb{R}^{d}} e^{2 \pi i x \cdot \xi} e^{-4 \pi^{2} i t|\xi|^{2}} \widehat{u_{0}}(\xi) d \xi,
$$

where the Fourier multiplier $e^{i t \Delta}$ is known as Schrödinger propagator. The corresponding inhomogeneous equation is

$$
\left\{\begin{array}{l}
i \partial_{t} u+\Delta u=F(t, x) \\
u(0, x)=u_{0}(x)
\end{array}\right.
$$

with $t>0$ and $x \in \mathbb{R}^{d}, d \geq 1$. By Duhamel's principle and (2), the integral version of (3) has the form

$$
u(t, x)=e^{i t \Delta} u_{0}(\cdot)+\int_{0}^{t} e^{i(t-s) \Delta} F(s, \cdot) d s .
$$

The study of space-time integrability properties of the solution to (2) and (4) has been pursued by many authors in the last thirty years. The matter of fact is given by the Strichartz estimates, that have become a fundamental and amazing tool for the study of PDE's. They have been studied in the framework of different function/distribution spaces, like Lebesgue, Sobolev, Wiener amalgam and modulation spaces and have found applications to well-posedness and scattering theory for nonlinear Schrödinger equations [3, 8, 9, 11, 19, 24, 26, 36, 38, $39,49]$.

In this paper we exhibit these problems. First, in Section 3, we introduce the dispersive estimates and show how can be carried out for these different spaces. The classical $L^{p}$ dispersive estimates read as follows

$$
\left\|e^{i t \Delta} u_{0}\right\|_{L_{x}^{r}} \lesssim|t|^{-d\left(\frac{1}{2}-\frac{1}{r}\right)}\left\|u_{0}\right\|_{L_{x}^{r^{\prime}}}, \quad 2 \leq r \leq \infty, \quad \frac{1}{r}+\frac{1}{r^{\prime}}=1 .
$$

Section 4 is devoted to the study of Strichartz estimates. The nature of these estimates is highlighted and the results among different kinds of spaces are compared with each others. Historically, the $L^{p}$ spaces $[19,24,26,39,49]$ were the first to be looked at. The celebrated homogeneous Strichartz estimates for the solution $u(t, x)=\left(e^{i t \Delta} u_{0}\right)(x)$ read

$$
\left\|e^{i t \Delta} u_{0}\right\|_{L_{t}^{q} L_{x}^{r}} \lesssim\left\|u_{0}\right\|_{L_{x}^{2}},
$$


for $q \geq 2, r \geq 2$, with $2 / q+d / r=d / 2,(q, r, d) \neq(2, \infty, 2)$, i.e., for $(q, r)$ Schrödinger admissible (see Definition 4.1). Here, as usual, we set

$$
\|F\|_{L_{t}^{q} L_{x}^{r}}=\left(\int\|F(t, \cdot)\|_{L_{x}^{r}}^{q} d t\right)^{1 / q} .
$$

In the sequel, the estimates for Sobolev spaces were essentially derived from the Lebesgue ones. Recently, several authors $([1,2,6,7,8,44,45])$ have turned their attention to fixed time and space-time estimates for the Schrödinger propagator between spaces widely used in time-frequency analysis, known as Wiener amalgam spaces and modulation spaces. The first appearance of amalgam spaces can be traced to Wiener in his development of the theory of generalized harmonic analysis [46, 47, 48] (see [22] for more details). In this setting, Cordero and Nicola $[6,7,8]$ have discovered that the pattern to obtain dispersive and Strichartz estimates is similar to that of Lebesgue spaces. The main idea is to show that the fundamental solution $K_{t}$ (see (20) below) lies in the Wiener amalgam space $W\left(L^{p}, L^{q}\right)$ (see Section 2 for the definition) which generalizes the classical $L^{p}$ space and, consequently, provides a different information between the local and global behavior of the solutions. Beside the similar arguments, we point out also some differences, mainly in proving the sharpness of dispersive estimates and Strichartz estimates. Indeed, dilation arguments in Wiener amalgam and modulation spaces don't work as in the classical $L^{p}$ spaces.

Modulation spaces were introduced by Feitchinger in 1980 and then were also redefined by Wang [44] using isometric decompositions. The two different definitions allow to look at the problem in two different manners. As a result, in [45], a beautiful use of interpolation theory on modulation spaces allows to combine the estimates obtained by means of the classical definition in $[1,2]$ and the isometric definition in $[44,45]$, to obtain more general fixed time estimates in this framework. In order to control the growth of singularity at $t=0$, we usually have the restriction $d(1 / 2-1 / p) \leqslant 1$; cf. [5, 26]. By using the isometric decomposition in the frequency space, as in $[44,45]$, one can remove the singularity at $t=0$ and preserve the decay at $t=\infty$ in certain modulation spaces.

The Strichartz estimates can be applied, e.g., to the well-posedness of non-linear Schrödinger equations or of linear Schrödinger equations with time-dependent potentials. We shall show examples in the last Section 5.

Notation. We define $|x|^{2}=x \cdot x$, for $x \in \mathbb{R}^{d}$, where $x \cdot y=x y$ is the inner product on $\mathbb{R}^{d}$. The space of smooth functions with compact support is denoted by $\mathscr{C}_{0}^{\infty}\left(\mathbb{R}^{d}\right)$, the Schwartz class by $\mathscr{S}\left(\mathbb{R}^{d}\right)$, the space of tempered distributions by $\mathscr{S}^{\prime}\left(\mathbb{R}^{d}\right)$. The Fourier transform is normalized to be $\hat{f}(\xi)=\mathscr{F} f(\xi)=\int f(t) e^{-2 \pi i t \xi} d t$. Translation and modulation operators (time and frequency shifts) are defined, respectively, by

$$
T_{x} f(t)=f(t-x) \text { and } M_{\xi} f(t)=e^{2 \pi i \xi t} f(t) .
$$


We have the formulas $\left(T_{x} f\right)^{\gamma}=M_{-x} \hat{f},\left(M_{\xi} f\right)^{\gamma}=T_{\xi} \hat{f}$, and $M_{\xi} T_{x}=e^{2 \pi i x \xi} T_{x} M_{\xi}$. The notation $A \lesssim B$ means $A \leq c B$ for a suitable constant $c>0$, whereas $A=B$ means $c^{-1} A \leq B \leq c A$, for some $c \geq 1$. The symbol $B_{1} \hookrightarrow B_{2}$ denotes the continuous embedding of the linear space $B_{1}$ into $B_{2}$.

\section{Function Spaces and Preliminaries}

In this section we present the function/distribution spaces we work with, and the properties used in our study.

\subsection{Lorentz spaces}

([34, 35]). We recall that the Lorentz space $L^{p, q}$ on $\mathbb{R}^{d}$ is defined as the space of tempered distributions $f$ such that

$$
\|f\|_{p q}^{*}=\left(\frac{q}{p} \int_{0}^{\infty}\left[t^{1 / p} f^{*}(t)\right]^{q} \frac{d t}{t}\right)^{1 / q}<\infty
$$

when $1 \leq p<\infty, 1 \leq q<\infty$, and

$$
\|f\|_{p q}^{*}=\sup _{t>0} t^{1 / p} f^{*}(t)<\infty
$$

when $1 \leq p \leq \infty, q=\infty$. Here, as usual, $\lambda(s)=|\{|f|>s\}|$ denotes the distribution function of $f$ and $f^{*}(t)=\inf \{s: \lambda(s) \leq t\}$.

One has $L^{p, q_{1}} \hookrightarrow L^{p, q_{2}}$ if $q_{1} \leq q_{2}$, and $L^{p, p}=L^{p}$. Moreover, for $1<p<\infty$ and $1 \leq q \leq \infty$, $L^{p, q}$ is a normed space and its norm $\|\cdot\|_{L^{p, q}}$ is equivalent to the above quasi-norm $\|\cdot\|_{p q}^{*}$.

The function $|x|^{-\alpha}$ lives in $L^{d / \alpha, \infty}, 0<\alpha<d$ but observe that this function doesn't live in any $L^{p}, 1 \leqslant p \leqslant \infty$. We now recall the following classical Hardy-Littlewood-Sobolev fractional integration theorem (see e.g. [33, Theorem 1, pag 119] and [34]), which will be used in the sequel

Proposition 2.1. Let $d \geqslant 1,0<\alpha<d$ and $1<p<q<\infty$ such that

$$
\frac{1}{q}=\frac{1}{p}-\frac{d-\alpha}{d}
$$

Then the following estimate

$$
\left\||\cdot|^{-\alpha} * f\right\|_{q} \lesssim\|f\|_{p}
$$

holds for all $f \in L^{p}\left(\mathbb{R}^{d}\right)$. 
Potential and Sobolev spaces. For $s \in \mathbb{R}$, we define the Fourier multipliers $\langle\Delta\rangle^{s} f=$ $\mathscr{F}^{-1}\left(\left(1+|\cdot|^{2}\right)^{s / 2} \hat{f}\right)$, and $|\Delta|^{s} f=\mathscr{F}^{-1}\left(|\cdot|^{s} \hat{f}\right)$. Then, for $1 \leq p \leq \infty$, the potential space [4] is defined by

$$
W_{s}^{p}=\left\{f \in \mathscr{S}^{\prime},\langle\Delta\rangle^{s} f \in L^{p}\right\}
$$

with norm $\|f\|_{W_{s}^{p}}=\left\|\langle\Delta\rangle^{s} f\right\|_{L^{p}}$. The homogeneous potential space [4] is defined by

$$
\dot{W}_{s}^{p}=\left\{f \in \mathscr{S}^{\prime},|\langle\Delta\rangle|^{s} f \in L^{p}\right\}
$$

with norm $\|f\|_{\dot{W}_{s}^{p}}=\left\||\Delta|^{s} f\right\|_{L^{p}}$.

For $p=2$ the previous spaces are called Sobolev spaces $H_{s}^{p}$ and homogeneous Sobolev spaces $\dot{H}_{s}^{p}$, respectively.

\subsection{Wiener amalgam spaces}

([12, 14, 15, 16, 17]). Let $g \in \mathscr{C}_{0}^{\infty}$ be a test function that satisfies $\|g\|_{L^{2}}=1$. We will refer to $g$ as a window function. For $1 \leq p \leq \infty$, recall the $\mathscr{F} L^{p}$ spaces, defined by

$$
\mathscr{F} L^{p}\left(\mathbb{R}^{d}\right)=\left\{f \in \mathscr{S}^{\prime}\left(\mathbb{R}^{d}\right): \exists h \in L^{p}\left(\mathbb{R}^{d}\right), \hat{h}=f\right\} ;
$$

they are Banach spaces equipped with the norm

$$
\|f\|_{\mathscr{F} L^{p}}=\|h\|_{L^{p}}, \quad \text { with } \hat{h}=f .
$$

In the same way, for $1<p<\infty, 1 \leq q \leq \infty$, the Banach spaces $\mathscr{F} L^{p, q}$ are defined by

$$
\mathscr{F} L^{p, q}\left(\mathbb{R}^{d}\right)=\left\{f \in \mathscr{S}^{\prime}\left(\mathbb{R}^{d}\right): \exists h \in L^{p, q}\left(\mathbb{R}^{d}\right), \hat{h}=f\right\} ;
$$

equipped with the norm

$$
\|f\|_{\mathscr{F} L^{p, q}}=\|h\|_{L^{p, q}}, \quad \text { with } \hat{h}=f .
$$

Let $B$ one of the following Banach spaces: $L^{p}, \mathscr{F} L^{p}, 1 \leq p \leq \infty, \mathscr{F} L^{p, q}, 1<p<\infty, 1 \leq q \leq \infty$, valued in a Banach space, or also spaces obtained from these by real or complex interpolation. Let $C$ be the $L^{p}$ space, $1 \leq p \leq \infty$, scalar-valued. For any given function $f$ which is locally in $B$ (i.e. $g f \in B, \forall g \in \mathscr{C}_{0}^{\infty}$ ), we set $f_{B}(x)=\left\|f T_{x} g\right\|_{B}$.

The Wiener amalgam space $W(B, C)$ with local component $B$ and global component $C$ is defined as the space of all functions $f$ locally in $B$ such that $f_{B} \in C$. Endowed with the norm $\|f\|_{W(B, C)}=\left\|f_{B}\right\|_{C}, W(B, C)$ is a Banach space. Moreover, different choices of $g \in \mathscr{C}_{0}^{\infty}$ generate the same space and yield equivalent norms.

If $B=\mathscr{F} L^{1}$ (the Fourier algebra), the space of admissible windows for the Wiener amalgam spaces $W\left(\mathscr{F} L^{1}, C\right)$ can be enlarged to the so-called Feichtinger algebra $W\left(\mathscr{F} L^{1}, L^{1}\right)$. Recall that the Schwartz class $\mathscr{S}$ is dense in $W\left(\mathscr{F} L^{1}, L^{1}\right)$. 
We use the following definition of mixed Wiener amalgam norms. Given a measurable function $F$ of the two variables $(t, x)$ we set

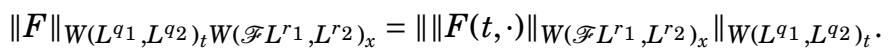

Observe that [6]

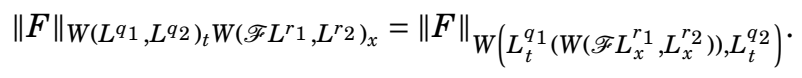

The following properties of Wiener amalgam spaces will be frequently used in the sequel.

Lemma 2.1. Let $B_{i}, C_{i}, i=1,2,3$, be Banach spaces such that $W\left(B_{i}, C_{i}\right)$ are well defined. Then,

(i) Convolution. If $B_{1} * B_{2} \hookrightarrow B_{3}$ and $C_{1} * C_{2} \hookrightarrow C_{3}$, we have

$$
W\left(B_{1}, C_{1}\right) * W\left(B_{2}, C_{2}\right) \hookrightarrow W\left(B_{3}, C_{3}\right) .
$$

In particular, for every $1 \leq p, q \leq \infty$, we have

$$
\|f * u\|_{W\left(\mathscr{F} L^{p}, L^{q}\right)} \leq\|f\|_{W\left(\mathscr{F} L^{\infty}, L^{1}\right)}\|u\|_{W\left(\mathscr{F} L^{p}, L^{q}\right)} .
$$

(ii) Inclusions. If $B_{1} \hookrightarrow B_{2}$ and $C_{1} \hookrightarrow C_{2}$,

$$
W\left(B_{1}, C_{1}\right) \hookrightarrow W\left(B_{2}, C_{2}\right) .
$$

Moreover, the inclusion of $B_{1}$ into $B_{2}$ need only hold "locally" and the inclusion of $C_{1}$ into $C_{2}$ "globally". In particular, for $1 \leq p_{i}, q_{i} \leq \infty, i=1,2$, we have

$$
p_{1} \geq p_{2} \text { and } q_{1} \leq q_{2} \Longrightarrow W\left(L^{p_{1}}, L^{q_{1}}\right) \hookrightarrow W\left(L^{p_{2}}, L^{q_{2}}\right) .
$$

(iii) Complex interpolation. For $0<\theta<1$, we have

$$
\left[W\left(B_{1}, C_{1}\right), W\left(B_{2}, C_{2}\right)\right]_{[\theta]}=W\left(\left[B_{1}, B_{2}\right]_{[\theta]},\left[C_{1}, C_{2}\right]_{[\theta]}\right),
$$

if $C_{1}$ or $C_{2}$ has absolutely continuous norm.

(iv) Duality. If $B^{\prime}, C^{\prime}$ are the topological dual spaces of the Banach spaces $B, C$ respectively, and the space of test functions $\mathscr{C}_{0}^{\infty}$ is dense in both $B$ and $C$, then

$$
W(B, C)^{\prime}=W\left(B^{\prime}, C^{\prime}\right) .
$$

The proof of all these results can be found in ([12, 14, 15, 22]).

Finally, let us recall the following lemma [8, Lemma 6.1], that will be used in the last Section 5 . 
Lemma 2.2. Let $1 \leq p, q, r \leq \infty$. If

$$
\frac{1}{p}+\frac{1}{q}=\frac{1}{r^{\prime}}
$$

then

$$
W\left(\mathscr{F} L^{p^{\prime}}, L^{p}\right)\left(\mathbb{R}^{d}\right) \cdot W\left(\mathscr{F} L^{q \prime}, L^{q}\right)\left(\mathbb{R}^{d}\right) \subset W\left(\mathscr{F} L^{r}, L^{r^{\prime}}\right)\left(\mathbb{R}^{d}\right)
$$

with norm inequality $\|f h\|_{W\left(\mathscr{F} L^{r}, L^{r^{\prime}}\right)} \lesssim\|f\|_{W\left(\mathscr{F} L^{\left.p^{\prime}, L^{p}\right)}\right.}\|h\|_{W\left(\mathscr{F} L^{q^{\prime}}, L^{q}\right)}$.

\subsection{Modulation spaces}

([13, 21]). Let $g \in \mathscr{S}\left(\mathbb{R}^{d}\right)$ be a non-zero window function and consider the so-called short-time Fourier transform (STFT) $V_{g} f$ of a function/tempered distribution $f$ with respect to the the window $g$ :

$$
V_{g} f(x, \xi)=\left\langle f, M_{\xi} T_{x} g\right\rangle=\int e^{-2 \pi i \xi y} f(y) \overline{g(y-x)} d y,
$$

i.e., the Fourier transform $\mathscr{F}$ applied to $f \overline{T_{x} g}$.

For $s \in \mathbb{R}$, we consider the weight function $\langle x\rangle^{s}=\left(1+|x|^{2}\right)^{s / 2}, x \in \mathbb{R}^{d}$. If $1 \leq p, q \leq \infty, s \in \mathbb{R}$, the modulation space $\mathscr{M}_{s}^{p, q}\left(\mathbb{R}^{d}\right)$ is defined as the closure of the Schwartz class with respect to the norm

$$
\|f\|_{\mathcal{M}_{s}^{p, q}}=\left(\int_{\mathbb{R}^{d}}\left(\int_{\mathbb{R}^{d}}\left|V_{g} f(x, \xi)\right|^{p} d x\right)^{q / p}\langle\xi\rangle^{s q} d \xi\right)^{1 / q}
$$

(with obvious modifications when $p=\infty$ or $q=\infty$ ).

Among the properties of modulation spaces, we record that they are Banach spaces whose

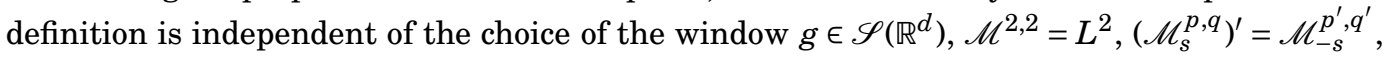
whenever $p, q<\infty$.

Another definition of these spaces uses the unite-cube decomposition of the frequency space, we address interested readers to [44].

Finally we recall the behaviour of modulation spaces with respect to complex interpolation (see [14, Corollary 2.3]).

Proposition 2.2. Let $1 \leq p_{1}, p_{2}, q_{1}, q_{2} \leq \infty$, with $q_{2}<\infty$. If $T$ is a linear operator such that, for $i=1,2$,

$$
\|T f\|_{M^{p_{i}, q_{i}}} \leq A_{i}\|f\|_{M^{p_{i}, q_{i}}} \quad \forall f \in M^{p_{i}, q_{i}},
$$

then

$$
\|T f\|_{M^{p, q}} \leq C A_{1}^{1-\theta} A_{2}^{\theta}\|f\|_{M^{p, q}} \quad \forall f \in M^{p, q},
$$

where $1 / p=(1-\theta) / p_{1}+\theta / p_{2}, 1 / q=(1-\theta) / q_{1}+\theta / q_{2}, 0<\theta<1$ and $C$ is independent of $T$.

We observe that definition and properties of modulation spaces refer to the case $p, q \geqslant 1$. For the quasi-Banach case $0<p, q<1$ see, e.g., [2, 44, 45]. 


\section{$2.4 T^{*} T$ method}

$[19,20]$ The $T^{*} T$ method is an abstract tool of Harmonic Analysis, discovered by Tomas in 1975. This method allows to know the continuity of a linear operator $T$ (and thus of its adjoint $T^{*}$ ), simply by the boundedness of the composition operator $T^{*} T$.

For any vector space $D$, we denote by $D_{a}^{*}$ its algebraic dual, by $\mathscr{L}_{a}(D, X)$ the space of linear maps from $D$ to some other vector space $X$, and by $\langle\varphi, f\rangle_{D}$ the pairing between $D_{a}^{*}$ and $D\left(f \in D, \varphi \in D_{a}^{*}\right)$, taken to be linear in $f$ and antilinear in $\varphi$.

Lemma 2.3. Let $\mathscr{H}$ be a Hilbert space, $X$ a Banach space, $X^{*}$ the dual of $X$, and $D$ a vector space densely contained in $X$. Let $T \in \mathscr{L}_{a}(D, \mathscr{H})$ and $T^{*} \in \mathscr{L}_{a}\left(\not{H}, D_{a}^{*}\right)$ be its adjoint, defined by

$$
\left\langle T^{*} h, f\right\rangle_{D}=\langle h, T f\rangle, \quad \forall f \in D, \quad \forall h \in \mathscr{H},
$$

where $\langle$,$\rangle is the inner product in \mathscr{H}$ (antilinear in the first argument). Then the following three conditions are equivalent.

(1) There exists $a, 0 \leqslant a<\infty$ such that for all $f \in D$

$$
\|T f\|_{\mathscr{H}} \leqslant a\|f\|_{X}
$$

(2) Let $h \in \mathcal{H}$. Then $T^{*} h$ can be extended to a continuous linear functional on $X$, and there exists $a, 0 \leqslant a<\infty$, such that for all $h \in \mathscr{H}$

$$
\left\|T^{*} h\right\|_{X^{*}} \leqslant a\|h\|_{\mathscr{H}}
$$

(3) Let $f \in X$. Then $T^{*} T f$ can be extended to a continuous linear functional on $X$, and there exists $a, 0 \leqslant a<\infty$, such that for all $f \in D$,

$$
\left\|T^{*} T f\right\|_{X^{*}} \leqslant a^{2}\|f\|_{X} .
$$

The constant a is the same in all the three cases. If one of (all) those conditions is (are) satisfied, the operators $T$ and $T^{*} T$ extend by continuity to bounded operators from $X$ to $\mathscr{H}$ and from $X$ to $X^{*}$, respectively.

Proof. From the fact that $D$ is densely contained in $X$, it follows that $X^{*}$ is a subspace of $D_{a}^{*}$. $(1) \Rightarrow(2)$. Let $h \in \mathcal{H}$. Then, for all $f \in D$

$$
\left|\left\langle T^{*} h, f\right\rangle_{D}\right|=|\langle h, T f\rangle| \leqslant\|h\|_{\mathscr{H}}\|T f\|_{\mathscr{H}} \leqslant a\|h\|_{\mathscr{H}}\|f\|_{X} .
$$

(2) $\Rightarrow(1)$. Let $f \in D$. Then, for all $h \in \mathscr{H}$

$$
|\langle h, T f\rangle|=\left|\left\langle T^{*} h, f\right\rangle_{D}\right| \leqslant\left\|T^{*} h\right\|_{X^{*}}\|f\|_{X} \leqslant a\|h\|_{\mathscr{H}}\|f\|_{X}
$$


Clearly (1) and (2) imply (3), and therefore (1) or (2) implies (3).

(3) $\Rightarrow(1)$. Let $f \in D$. Then

$$
\|T f\|^{2}=|\langle T f, T f\rangle|=\left|\left\langle T^{*} T f, f\right\rangle_{D}\right| \leqslant\left\|T^{*} T f\right\|_{X^{*}}\|f\|_{X} \leqslant a^{2}\|f\|_{X}^{2} .
$$

Since $D$ is a dense subspace of $X$, we see that $T$ can be extended to a bounded linear functional from $X$ to $\mathscr{H}$.

The following corollary is extremely useful.

Corollary 2.3. Let $\mathscr{H}, \mathscr{D}$ and two triplets $\left(X_{i}, T_{i}, a_{i}\right), i=1,2$, satisfy the conditions of Lemma 2.3. Then for all choices of $i, j=1,2, \mathscr{R}\left(T_{i}^{*} T_{j}\right) \subset X_{i}^{*}$ and for all $f \in D$,

$$
\left\|T_{i}^{*} T_{j} f\right\|_{X_{i}^{*}} \leqslant a_{i} a_{j}\|f\|_{X_{j}} .
$$

In particular, $T_{i}^{*} T_{j}$ extends by continuity to a bounded operator from $X_{j}$ to $X_{i}^{*}$, and (17) holds for all $f \in X_{j}$.

Ginibre and Velo [19] applied Lemma 2.3 and Corollary 2.3 to the bounded operator $T: L^{1}(I, \mathscr{H}) \rightarrow \mathscr{H}$, defined by

$$
T f=\int_{I} U(-t) f(t) d t
$$

where $I$ is an interval of $\mathbb{R}$ (possibly $\mathbb{R}$ itself) and $U$ a unitary strongly continuous one parameter group in $\mathscr{H}$. Then its adjoint $T^{*}$ is the operator

$$
T^{*} h(t)=U(t) h
$$

from $\mathscr{H}$ to $L^{\infty}(I, \mathscr{H})$, where the duality is defined by the scalar products in $\mathscr{H}$ and in $L^{2}(I, \mathscr{C})$, such that $T^{*} T$ is the bounded operator from $L^{1}(I, \mathscr{H})$ to $L^{\infty}(I, \mathscr{H})$ given by

$$
T^{*} T f=\int_{I} U\left(t-t^{\prime}\right) f\left(t^{\prime}\right) d t^{\prime} .
$$

Clearly the conditions of Lemma 2.3 are satisfied with $X=L^{1}(I, \mathscr{H})$, the operator $T$ defined in (18), the constant $a=1$, and $\mathscr{D}$ any dense subspace of $X$.

Let us introduce the retarded operator $\left(T^{*} T\right)_{R}$, defined by

$$
\left(T^{*} T\right)_{R} f(t)=\left(U_{R} *_{t} f\right)(t)=\int_{I} U_{R}\left(t-t^{\prime}\right) f\left(t^{\prime}\right) d t^{\prime}
$$

where $U_{R}(t)=\chi_{+}(t) U(t):=\chi_{[0, \infty)}(t) U(t)$.

We recall that a space $X$ of distributions in space-time is said to be time cut-off stable if the multiplication by the characteristic function $\chi_{J}$, of an interval $J$ in time, is a bounded operator in $X$ with norm uniformly bounded with respect to $J$. The spaces under our consideration are of the type $X=L_{t}^{q}(I, Y)$, where $Y$ is a space of distribution in the space variable and for which that property obviously holds. 
Lemma 2.4. Let $\mathscr{H}$ an Hilbert space, let I be an interval of $\mathbb{R}$, let $X \subset \mathscr{S}^{\prime}\left(I \times \mathbb{R}^{d}\right)$ be a Banach space, let $X$ be time cut-off stable, and let the conditions of Lemma 2.3 hold for the operator $T$ defined in (18). Then the operator $\left(T^{*} T\right)_{R}$ is (strictly speaking extends to) a bounded operator from $L_{t}^{1}(I, \mathscr{H})$ to $X^{*}$ and from $X$ to $L_{t}^{\infty}(I, \mathscr{H})$.

Proof. We recall the proof for sake of clarity. It is enough to demonstrate the second property, from which the first one follows by duality. Let $f \in D$. Then, for each $t$

$$
\begin{aligned}
\left\|\left(T^{*} T\right)_{R} f(t)\right\|_{\mathscr{H}} & =\left\|T \chi_{+}(t-\cdot) f\right\|_{\mathscr{H}} \leqslant a \sup _{t}\left\{\left\|\chi_{+}(t-\cdot)\right\|_{\mathscr{B}(X)}\right\}\|f\|_{X} \\
& \leq C a\|f\|_{X},
\end{aligned}
$$

by the unitary of $U$, the estimate (14) of Lemma 2.3, and the time cut-off stability of $X$.

\section{$3 \quad$ Fixed Time Estimates}

In this section we study estimates for the solution $u(t, x)$ to the Cauchy problem (1), for fixed $t$. Since multiplication on the Fourier transform side intertwines with convolution on the space side, formula (2) can be rewritten as

$$
u(t, x)=\left(K_{t} * u_{0}\right)(x),
$$

where $K_{t}$ is the inverse Fourier transform of the multiplier $e^{-4 \pi^{2} i t|\xi|^{2}}$, given by

$$
K_{t}(x)=\frac{1}{(4 \pi i t)^{d / 2}} e^{i|x|^{2} /(4 t)}
$$

First, we establish the estimates for Lebesgue spaces. Since $e^{i t \Delta}$ is a unitary operator, we obtain the $L^{2}$ conservation law

$$
\left\|e^{i t \Delta} u_{0}\right\|_{L^{2}\left(\mathbb{R}^{d}\right)}=\left\|u_{0}\right\|_{L^{2}\left(\mathbb{R}^{d}\right)} .
$$

Furthermore, since $K_{t} \in L^{\infty}$ with $\left\|K_{t}\right\|_{\infty}=t^{-d / 2}$, applying Young inequality to the fundamental solution (19) we obtain the $L^{1}$ dispersive estimate

$$
\left\|e^{i t \Delta} u_{0}\right\|_{L^{\infty}\left(\mathbb{R}^{d}\right)} \lesssim|t|^{-d / 2}\left\|u_{0}\right\|_{L^{1}\left(\mathbb{R}^{d}\right)} .
$$

This shows that if the initial data $u_{0}$ has a suitable integrability in space, then the evolution will have a power-type decay in time. Using the Riesz-Thorin theorem (see, e.g., [35]), we can interpolate (21) and (22) to obtain the important $L^{p}$ fixed time estimates

$$
\left\|e^{i t \Delta} u_{0}\right\|_{L^{r}\left(\mathbb{R}^{d}\right)} \lesssim|t|^{-d\left(\frac{1}{2}-\frac{1}{r}\right)}\left\|u_{0}\right\|_{L^{r^{\prime}\left(\mathbb{R}^{d}\right)}}
$$


for all $2 \leqslant r \leqslant \infty$, with $1 / r+1 / r^{\prime}=1$. These estimates represent the complete range of $L^{p}$ to $L^{q}$ fixed time estimates available. In this setting, the necessary conditions are usually obtained by scaling conditions (see, for example, [39, Exercise 2.35], and [29] for the interpretation in terms of Gaussian curvature of the characteristic manifold). The following proposition ([50, page 45]) is an example of this technique in the case $p=q^{\prime}$.

Proposition 3.1. Let $1 \leqslant r \leqslant \infty$ and $\alpha \in \mathbb{R}$ such that

$$
\left\|e^{i t \Delta} u_{0}\right\|_{L^{r}\left(\mathbb{R}^{d}\right)} \leqslant C t^{\alpha}\left\|u_{0}\right\|_{L^{r^{\prime}\left(\mathbb{R}^{d}\right)}},
$$

for all $u_{0} \in S\left(\mathbb{R}^{d}\right), t \neq 0$ and some $C$ independent of $t$ and $u_{0}$. Then $\alpha=-d\left(\frac{1}{2}-\frac{1}{r}\right), r^{\prime} \leqslant r$ (and thus $2 \leqslant r \leqslant \infty$ ).

Proof. We can rescale the initial data $u_{0}$ by a factor $\lambda$ and use (24) for

$$
v(x):=u_{0}(\lambda x), \quad \lambda>0, \quad u_{0} \in \mathscr{S}\left(\mathbb{R}^{d}\right) .
$$

The corresponding solution with $v(x)$ as initial data is $u\left(\lambda^{2} t, \lambda x\right)$, where $u(t, x)=e^{i t \Delta} u_{0}$. Therefore, by (24) and the scaling property

$$
\|f(\lambda \cdot)\|_{r}=\lambda^{-d / r}\|f(\cdot)\|_{r}
$$

one has

$$
\lambda^{-d / r}\left\|u\left(\lambda^{2} t, \cdot\right)\right\|_{L^{r}\left(\mathbb{R}^{d}\right)} \leqslant C t^{\alpha} \lambda^{-d / r^{\prime}}\left\|u_{0}\right\|_{L^{r^{\prime}\left(\mathbb{R}^{d}\right)}},
$$

for all $\lambda>0, t \neq 0$ and $u_{0} \in S\left(\mathbb{R}^{d}\right)$. Choosing $t=\lambda^{-2}$, we obtain

$$
\|u(1, \cdot)\|_{L^{r}\left(\mathbb{R}^{d}\right)} \leqslant C \lambda^{-2 \alpha-\frac{d}{r^{\prime}}+\frac{d}{r}}\left\|u_{0}\right\|_{L^{r^{\prime}\left(\mathbb{R}^{d}\right)}},
$$

for all $\lambda>0$ and $u_{0} \in S\left(\mathbb{R}^{d}\right)$. Since $\|u(1, \cdot)\|_{L^{r}\left(\mathbb{R}^{d}\right)}$ and $\left\|u_{0}\right\|_{L^{r^{\prime}\left(\mathbb{R}^{d}\right)}}$ are two positive constants, we have

$$
\begin{array}{ll}
\text { for } \lambda \rightarrow \infty, & -2 \alpha-\frac{d}{r^{\prime}}+\frac{d}{r} \geqslant 0, \\
\text { for } \lambda \rightarrow 0, & -2 \alpha-\frac{d}{r^{\prime}}+\frac{d}{r} \leqslant 0
\end{array}
$$

and then we obtain the necessary condition for $\alpha$. Moreover, since $e^{i t \Delta}$ is invariant under translation, by [23, Theorem 1.1] we obtain $r^{\prime} \leqslant r$, i.e., $2 \leqslant r \leqslant \infty$. By standard density argument we attain the desired result.

For $s \in \mathbb{R}$, consider the Fourier multiplier $\langle\Delta\rangle^{s}$, defined by $\langle\Delta\rangle^{s} f=\mathscr{F}^{-1}\left(\langle\cdot\rangle^{s} \hat{f}\right)$. Then, from (23) and the commutativity property of Fourier multipliers, one immediately obtains the $W^{s, r}$ fixed time estimates

$$
\left\|e^{i t \Delta} u_{0}\right\|_{W^{s, r}\left(\mathbb{R}^{d}\right)} \lesssim|t|^{-d\left(\frac{1}{2}-\frac{1}{r}\right)}\left\|u_{0}\right\|_{W^{s, r^{\prime}}\left(\mathbb{R}^{d}\right)}
$$


for all $s \in \mathbb{R}, 2 \leqslant r \leqslant \infty, 1 / r+1 / r^{\prime}=1$. Finally, we note that the conservation law (21) can be rephrased in this setting as the $H^{s}$ conservation law

$$
\left\|e^{i t \Delta} u_{0}\right\|_{H^{s}\left(\mathbb{R}^{d}\right)}=\left\|u_{0}\right\|_{H^{s}\left(\mathbb{R}^{d}\right)} .
$$

The Schrödinger propagator does not preserve any $W^{s, r}$ norm other than the $H^{s}$ norm.

Now, we focus on Wiener amalgam spaces. $K_{t}$ in (20) lives in $W\left(\mathscr{F} L^{1}, L^{\infty}\right) \subset L^{\infty}$, see $[1,6,44]$. This is the finest Wiener amalgam space-norm for $K_{t}$ which, consequently, gives the worst behavior in the time variable. It is also possible to improve the latter, at the expense of a rougher $x$-norm, see [8]. Indeed, since $K_{t} \in W\left(\mathscr{F} L^{p}, L^{\infty}\right)$ with norm (see [8, Corollary 3.1])

$$
\left\|K_{t}\right\|_{W\left(\mathscr{F} L^{p}, L^{\infty}\right)}=|t|^{-d / p}\left(1+t^{2}\right)^{(d / 2)(1 / p-1 / 2)},
$$

from the fundamental solution (19) and the convolution relations for Wiener amalgam spaces in Lemma 2.1(i), it turns out, for $2 \leq q \leq \infty$, the $W\left(\mathscr{F} L^{p}, L^{q}\right)$ dispersive estimates

$$
\left\|e^{i t \Delta} u_{0}\right\|_{W\left(\mathscr{F} L^{q^{\prime}}, L^{\infty}\right)} \lesssim|t|^{d(2 / q-1)}\left(1+t^{2}\right)^{d(1 / 4-1 / q)}\left\|u_{0}\right\|_{W\left(\mathscr{F} L^{q}, L^{1}\right)} .
$$

As well as for Lebesgue spaces, we can use complex interpolation between the dispersive estimates (28) and the $L^{2}$ conservation law $\left(L^{2}=W\left(\mathscr{F} L^{2}, L^{2}\right)\right)$ to obtain the following $W\left(\mathscr{F} L^{p}, L^{q}\right)$ fixed time estimates, that combine [6, Theorem 3.5] and [8, Theorem 3.3].

Theorem 3.2. For $2 \leq q, r, s \leq \infty$ such that

$$
\frac{1}{s}=\frac{1}{r}+\frac{2}{q}\left(\frac{1}{2}-\frac{1}{r}\right),
$$

we have

$$
\left\|e^{i t \Delta} u_{0}\right\|_{W\left(\mathscr{F} L^{s^{\prime}}, L^{r}\right)} \lesssim|t|^{d\left(\frac{2}{q}-1\right)\left(1-\frac{2}{r}\right)}\left(1+t^{2}\right)^{d\left(\frac{1}{4}-\frac{1}{q}\right)\left(1-\frac{2}{r}\right)}\left\|u_{0}\right\|_{W\left(\mathscr{F} L^{s}, L^{r^{\prime}}\right)}
$$

In particular, for $s=2$,

$$
\left\|e^{i t \Delta} u_{0}\right\|_{W\left(L^{2}, L^{r}\right)} \lesssim\left(1+t^{2}\right)^{-\frac{d}{2}\left(\frac{1}{2}-\frac{1}{r}\right)}\left\|u_{0}\right\|_{W\left(L^{2}, L^{r^{\prime}}\right)},
$$

and, for $s=r$,

$$
\left\|e^{i t \Delta} u_{0}\right\|_{W\left(\mathscr{F} L^{\left.r^{\prime}, L^{r}\right)}\right.} \lesssim\left(|t|^{-2}+|t|^{-1}\right)^{d\left(\frac{1}{2}-\frac{1}{r}\right)}\left\|u_{0}\right\|_{W\left(\mathscr{F} L^{r}, L^{r^{\prime}}\right)} \cdot
$$

Proof. Let us sketch the proof for the sake of readers. Estimate (29) follow by complex interpolation between estimate (28), which corresponds to $r=\infty$, and (21), which corresponds to $r=2$.

Indeed, $L^{2}=W\left(\mathscr{F} L^{2}, L^{2}\right)=W\left(L^{2}, L^{2}\right)$. Using Lemma 2.1(iii), with $\theta=2 / r$ (observe that $0<$ $2 / r<1)$, and $1 / s^{\prime}=(1-2 / r) / q^{\prime}+(2 / r) / 2$, so that relation $(29)$ holds, we obtain

$$
\left[W\left(\mathscr{F} L^{q^{\prime}}, L^{\infty}\right), W\left(\mathscr{F} L^{2}, L^{2}\right)\right]_{[\theta]}=W\left(\left[\mathscr{F} L^{q^{\prime}}, \mathscr{F} L^{2}\right]_{[\theta]},\left[L^{\infty}, L^{2}\right]_{[\theta]}\right)
$$




$$
=W\left(\mathscr{F} L^{s^{\prime}}, L^{r}\right)
$$

and

$$
\begin{aligned}
{\left[W\left(\mathscr{F} L^{q}, L^{1}\right), W\left(\mathscr{F} L^{2}, L^{2}\right)\right]_{[\theta]} } & =W\left(\left[\mathscr{F} L^{q}, \mathscr{F} L^{2}\right]_{[\theta]},\left[L^{1}, L^{2}\right]_{[\theta]}\right) \\
& =W\left(\mathscr{F} L^{s}, L^{r^{\prime}}\right) .
\end{aligned}
$$

This yields the desired estimate (29).

Let us compare the previous results with the classical $L^{p}$ estimates. For $2 \leqslant r \leqslant \infty$, $\mathscr{F} L^{r^{\prime}} \hookrightarrow L^{r}$, and the inclusion relations for Wiener amalgam spaces (Lemma 2.1 (ii)) yield $W\left(\mathscr{F} L^{r^{\prime}}, L^{r}\right) \hookrightarrow W\left(L^{r}, L^{r}\right)=L^{r}$ and $L^{r^{\prime}}=W\left(L^{r^{\prime}}, L^{r^{\prime}}\right) \hookrightarrow W\left(\mathscr{F} L^{r}, L^{r^{\prime}}\right)$. Thereby the estimate (31) is an improvement of (23) for every fixed time $t \neq 0$, and also uniformly for $|t|>c>0$. Moreover, in [8] Cordero and Nicola proved that the range $r \geq 2$ in (31) is sharp, and the same for the decay $t^{-d\left(\frac{1}{2}-\frac{1}{r}\right)}$ at infinity and the bound $t^{-2 d\left(\frac{1}{2}-\frac{1}{r}\right)}$, when $t \rightarrow 0$.

Modulation spaces are new settings inherited by time-frequency analysis where the fixed time estimates recently have been studied, see [1, 2, 44, 45]. Here, instead of using the representation of the solution $u(t, x)$ in (19), the solution is written in the form of Fourier multiplier $e^{i t \Delta} u_{0}$ as in (2), see [1,2]. Indeed, a sufficient condition for the boundedness of a Fourier multiplier on modulation spaces is that its symbol is in $W\left(\mathscr{F} L^{1}, l^{\infty}\right)([1$, Lemma 8]). Moreover, the Schrödinger symbol $\sigma=e^{-i t|\xi|^{2}}$ lives in $W\left(\mathscr{F} L^{1}, l^{\infty}\right)$ and its norm is

$$
\begin{aligned}
\|\sigma\|_{W\left(\mathscr{F} L^{1}, l^{\infty}\right)} & =\sup _{x} \int_{\mathbb{R}^{d}}\left|V_{g} \sigma(x, \omega)\right| d \omega \\
& =\left(1+t^{2}\right)^{-d / 4} \int_{\mathbb{R}^{d}} e^{-\frac{\pi}{t^{2}+1}|\omega|^{2}} d \omega=\left(1+t^{2}\right)^{d / 4},
\end{aligned}
$$

where $g(\xi)=e^{-\pi|\xi|^{2}}$. Then, by [2, Lemma 2] (also for $s=0$ [1, Corollary 18]) one has that $e^{i t \Delta}$ extends to a bounded operator on $\mathscr{M}_{s}^{p, q}$, i.e., the $\mathscr{M}_{s}^{p, q}$ fixed time estimates

$$
\|u(t, x)\|_{\mathscr{M}_{s}^{p, q}} \lesssim(1+|t|)^{d / 2}\left\|u_{0}\right\|_{\mathscr{M}_{s}^{p, q}}
$$

for all $s \geqslant 0$ and $1 \leqslant p, q \leqslant \infty$. In particular, modulation space properties are preserved by the time evolution of the Schrödinger equation, in strong contrast with the case of Lebesgue spaces. Observe that (32), in the case $s=0$, was also obtained using isometric decompositions in [44]. Later, Wang, Zaho, Guo in [45] obtain the following fixed time estimates

$$
\|u(t, x)\|_{\mathscr{M}_{s}^{p, q}} \lesssim(1+|t|)^{-d(1 / 2-1 / p)}\left\|u_{0}\right\|_{\mathscr{M}_{s}^{p^{\prime}, q}},
$$

for all $s \in \mathbb{R}, 2 \leqslant p \leqslant \infty$ and $1 \leqslant q \leqslant \infty$. Comparing (23) with (32) and (33), we see that the singularity at $t=0$ contained in (23) has been removed in (32) and (33) and the decay rate in (33) when $t=\infty$ is the same one as in (23). The estimate (33) also indicates that $e^{i t \Delta}$ is 
uniformly bounded on $\mathscr{M}^{2, q}$. The complex interpolation between the case $p=2$ in (33), and $p=\infty$ in (32) yields

$$
\|u(t, x)\|_{\mathscr{M}_{s}^{p, q}} \lesssim(1+|t|)^{d(1 / 2-1 / p)}\left\|u_{0}\right\|_{\mathscr{M}_{s}^{p, q}}
$$

for all $2 \leqslant p \leqslant \infty, s \geqslant 0$. However, it is still not clear whether the growth order on time in the right-hand side of (34) is optimal.

\section{Strichartz Estimates}

In many applications, especially in the study of well-posedness of PDE's, it is useful to have estimates for the solution both in time and space variables. In this direction, the main result is represented by the Strichartz estimates. First, let us introduce the following definitions.

Definition 4.1. Following [26], we say that the exponent pair $(q, r)$ is Schrödinger-admissible if $d \geqslant 1$ and

$$
2 \leqslant q, r \leqslant \infty, \quad \frac{1}{q}=\frac{d}{2}\left(\frac{1}{2}-\frac{1}{r}\right), \quad(q, r, d) \neq(2, \infty, 2) .
$$

Definition 4.2. Following [18], we say that the exponent pair $(q, r)$ is Schrödinger-acceptable if

$$
1 \leqslant q<\infty, \quad 2 \leqslant r \leqslant \infty, \quad \frac{1}{q}<d\left(\frac{1}{2}-\frac{1}{r}\right), \text { or } \quad(q, r)=(\infty, 2)
$$

The original version of Strichartz estimates in $L^{p}$ spaces, closely related to restriction problem of Fourier transform to surfaces, was elaborated by Robert Strichartz [36] in 1977(who, in turn, had precursors in [31, 41]). In 1995 a brilliant idea of Ginibre and Velo [20] was the use of the $T^{*} T$ Method (Lemma 2.3) to detach the couple ( $\left.q, r\right)$ from $\left(q^{\prime}, r^{\prime}\right)$ (see also [49]). The study of the endpoint case $(q, r)=(2,2 d /(d-2))$ is treated in [26], where Keel and Tao prove the estimate also for the endpoint when $d \geq 3$ (for $d=2$, the endpoint is $(q, r)=(2, \infty)$ and the estimate is false). We shall give a standard proof of the $L^{p}$ Stichartz estimates in the nonendpoint cases [10, 50] (see also [39] where the following theorem is proved using an abstract lemma, the Christ-Kiselev Lemma, which is very useful in establishing retarded Strichartz estimates).

Theorem 4.3. For any Schrödinger-admissible couples $(q, r)$ and $(\tilde{q}, \tilde{r})$ one has the homogeneous Strichartz estimates

$$
\left\|e^{i t \Delta} u_{0}\right\|_{L_{t}^{q} L_{x}^{r}\left(\mathbb{R} \times \mathbb{R}^{d}\right)} \lesssim\left\|u_{0}\right\|_{L_{x}^{2}\left(\mathbb{R}^{d}\right)},
$$

the dual homogeneous Strichartz estimates

$$
\left\|\int_{\mathbb{R}} e^{-i s \Delta} F(s, \cdot) d s\right\|_{L_{x}^{2}\left(\mathbb{R}^{d}\right)} \lesssim\|F\|_{L_{t}^{\tilde{q}^{\prime}} L_{x}^{\tilde{r}^{\prime}}\left(\mathbb{R} \times \mathbb{R}^{d}\right)}
$$


and the inhomogenous (retarded) Strichartz estimates

$$
\left\|\int_{s<t} e^{i(t-s) \Delta} F(s, \cdot) d s\right\|_{L_{t}^{q} L_{x}^{r}\left(\mathbb{R} \times \mathbb{R}^{d}\right)} \lesssim\|F\|_{L_{t}^{\tilde{q}^{\prime}} L_{x}^{\tilde{r}^{\prime}\left(\mathbb{R} \times \mathbb{R}^{d}\right)^{\prime}}} .
$$

Proof. We shall only prove this theorem in the non-endpoint case, when $q \neq 2$, addressing the interested reader to [26] for the whole study. We use the $T^{*} T$ method as follows. Let $(q, r)$ be Schrödinger admissible and consider the linear operator $T: L_{t}^{1} L_{x}^{2} \longrightarrow L_{x}^{2}$, defined as

$$
T(F)=\int_{\mathbb{R}} e^{-i s \Delta} F(s, \cdot) d s .
$$

Its adjoint $T^{*}: L_{x}^{2} \longrightarrow L_{t}^{\infty} L_{x}^{2}$ is the Schrödinger propagator (2)

$$
T^{*}(u)=e^{i t \Delta} u .
$$

Applying Minkowski's inequality, the fixed time estimate (23) and (6), we obtain the diagonal untruncated estimates

$$
\begin{aligned}
& \left\|\int_{\mathbb{R}} e^{i(t-s) \Delta} F(s, \cdot) d s\right\|_{L_{t}^{q} L_{x}^{r}\left(\mathbb{R} \times \mathbb{R}^{d}\right)} \leqslant\left\|\int_{\mathbb{R}}\right\| e^{i(t-s) \Delta} F(s, \cdot)\left\|_{L_{x}^{r}\left(\mathbb{R}^{d}\right)} d s\right\|_{L_{t}^{q}(\mathbb{R})} \\
& \lesssim\|\| F\left\|_{L_{x}^{r^{\prime}\left(\mathbb{R}^{d}\right)}} * \frac{1}{|t|^{d\left(\frac{1}{2}-\frac{1}{r}\right)}}\right\|_{L_{t}^{q}(\mathbb{R})} \lesssim\|F\|_{L_{t}^{q^{\prime}} L_{x}^{r^{\prime}\left(\mathbb{R} \times \mathbb{R}^{d}\right)}},
\end{aligned}
$$

whenever $2<q, r \leqslant \infty$ are such that $\frac{2}{q}+\frac{d}{r}=\frac{d}{2}$, and for any Schwartz function $F \in \mathscr{S}(\mathbb{R} \times$ $\mathbb{R}^{d}$ ). Then, using Lemma 2.3, one obtains the homogeneous Strichartz estimates (35) and the corresponding dual homogeneous Strichartz estimates (36). Corollary 2.3 applied to the previous two estimates yields the non-diagonal untruncate estimates:

$$
\left\|\int_{\mathbb{R}} e^{i(t-s) \Delta} F(s, \cdot) d s\right\|_{L_{t}^{q} L_{x}^{r}\left(\mathbb{R} \times \mathbb{R}^{d}\right)} \leqslant\|F\|_{L_{t}^{\tilde{q}^{\prime}} L_{x}^{\tilde{r}^{\prime}}\left(\mathbb{R} \times \mathbb{R}^{d}\right)} .
$$

By untruncated diagonal estimates one obtains the diagonal ones for the truncated operator, noting that

$$
\begin{aligned}
\left\|\int_{-\infty}^{t} e^{i(t-s) \Delta} F(s, \cdot) d s\right\|_{L_{t}^{q} L_{x}^{r}\left(\mathbb{R} \times \mathbb{R}^{d}\right)} & \leqslant\left\|\int_{-\infty}^{t}\right\| e^{i(t-s) \Delta} F(s, \cdot)\left\|_{L_{x}^{r}\left(\mathbb{R}^{d}\right)} d s\right\|_{L_{t}^{q}(\mathbb{R})} \\
& \leqslant\left\|\int_{\mathbb{R}}\right\| e^{i(t-s) \Delta} F(s, \cdot)\left\|_{L_{x}^{r}\left(\mathbb{R}^{d}\right)} d s\right\|_{L_{t}^{q}(\mathbb{R})} \lesssim\|F\|_{L_{t}^{q^{\prime}} L_{x}^{r^{\prime}\left(\mathbb{R} \times \mathbb{R}^{d}\right)}} .
\end{aligned}
$$

Moreover, using Lemma 2.4, with $X=L_{t}^{q^{\prime}} L_{x}^{r^{\prime}}$ and the truncated operator $\left(T^{*} T\right)_{R} F(t)=\int_{0}^{t} e^{i(t-s) \Delta}$ $F(s) d s$, one obtains

$$
\left\|\int_{0}^{t} e^{i(t-s) \Delta} F(s, \cdot) d s\right\|_{L_{t}^{\infty} L_{x}^{2}\left(\mathbb{R} \times \mathbb{R}^{d}\right)} \lesssim\|F\|_{L_{t}^{q^{\prime}} L_{x}^{r^{\prime}}}
$$

for all admissible pairs $(q, r)$. Then, by complex interpolation between this estimate and the diagonal truncated ones above one gets the non-diagonal truncate estimates (37), for any couple $(q, r),(\tilde{q}, \tilde{r})$ Schrödinger admissible. 
The estimates are known to fail at the endpoint $(q, r, d)=(2, \infty, 2)$, see [28], where Smith constructed a counterexample using the Brownian motion, although the homogeneous estimates can be saved assuming spherical symmetry [27, 32, 38]. The exponents in the homogeneous estimates are optimal ([39, Exercise 2.42]); some additional estimates are instead available in the inhomogeneous case (see, for example, [30]). Indeed, Kato [25] proved that inhomogeneous estimates (37) hold true when the pairs $(q, r)$ and $(\tilde{q}, \tilde{r})$ are Schrödinger acceptable and satisfy the scaling condition $1 / q+1 / \tilde{q}=d / 2(1-1 / r-1 / \tilde{r})$ in the range $1 / r, 1 / \tilde{r}>(d-2) /(2 d)$. Afterwards, for $d>2$, Foschi [18] improved this result by looking for the optimal range of Lebesgue exponents for which inhomogeneous Strichartz estimates hold (results almost equivalent have recently obtained by Vilela [43]). Actually, this range is larger than the one given by admissible exponents for homogeneous estimates, as was shown by the following result [18, Proposition 24].

Proposition 4.4. If $v$ is the solution to (3), with zero initial data and inhomogeneous term $F$ supported on $\mathbb{R} \times \mathbb{R}^{d}$, then we have the estimate

$$
\|v\|_{L_{t}^{q} L_{x}^{r}\left(\mathbb{R} \times \mathbb{R}^{d}\right)} \lesssim\|F\|_{L_{t}^{\tilde{q}^{\prime}} L_{x}^{\tilde{r}^{\prime}}\left(\mathbb{R} \times \mathbb{R}^{d}\right)}
$$

whenever $(q, r),(\tilde{q}, \tilde{r})$ are Schrödinger acceptable pairs which satisfy the scaling condition

$$
\frac{1}{q}+\frac{1}{\tilde{q}}=\frac{d}{2}\left(1-\frac{1}{r}-\frac{1}{\tilde{r}}\right)
$$

and either the conditions

$$
\frac{1}{q}+\frac{1}{\tilde{q}}<1, \quad \frac{d-2}{r} \leqslant \frac{d}{\tilde{r}}, \quad \frac{d-2}{\tilde{r}} \leqslant \frac{d}{r}
$$

or the conditions

$$
\frac{1}{q}+\frac{1}{\tilde{q}}=1, \quad \frac{d-2}{r}<\frac{d}{\tilde{r}}, \quad \frac{d-2}{\tilde{r}}<\frac{d}{r}, \quad \frac{1}{r} \leqslant \frac{1}{q}, \quad \frac{1}{\tilde{r}} \leqslant \frac{1}{\tilde{q}} .
$$

For a discussion about the sharpness of this proposition we refer to [18], where explicit counterexamples are constructed to show the necessary conditions for inhomogeneous Strichartz estimates.

Since the Schrödinger operator $e^{i t \Delta}$ commutes with Fourier multipliers like $|\Delta|^{s}$ or $\langle\Delta\rangle^{s}$, it is easy to obtain Strichartz estimates for potential and Sobolev spaces. In particular, if $I$ is an interval containing the origin and $u: I \times \mathbb{R}^{d} \rightarrow \mathbb{C}$ is the solution to the inhomogeneous Schrödinger equation with initial data $u_{0} \in \dot{H}_{x}^{s}\left(\mathbb{R}^{d}\right)$, given by the Duhamel formula (4), then, applying $|\Delta|^{s}$ to both sides of the equation and using the estimate of Theorem 4.3, one obtains

$$
\|u\|_{L_{t}^{q} \dot{W}_{x}^{s, r}\left(I \times \mathbb{R}^{d}\right)} \lesssim\left\|u_{0}\right\|_{\dot{H}_{x}^{s}\left(\mathbb{R}^{d}\right)}+\|F\|_{L_{t}^{\tilde{q}^{\prime}} \dot{W}_{x}^{s, \tilde{r}^{\prime}}\left(I \times \mathbb{R}^{d}\right)}
$$


for all Schrödinger admissible couples $(q, r)$ and $(\tilde{q}, \tilde{r})$. In particular, if one considers the homogeneous case (i.e. $F=0$ ), the Sobolev embedding $\dot{W}_{x}^{s, r} \hookrightarrow L_{x}^{r_{1}}, 0<s<d / 2$ and $1 / r_{1}=$ $1 / r-s / d$, yields the $\dot{H}^{s}$ Stichartz estimates

$$
\|u\|_{L_{t}^{q} L_{x}^{r_{1}}\left(I \times \mathbb{R}^{d}\right)} \lesssim\left\|u_{0}\right\|_{\dot{H}_{x}^{s}\left(\mathbb{R}^{d}\right)}, \quad \frac{2}{q}+\frac{d}{r_{1}}+s=\frac{d}{2}
$$

Since $s>0$ one has

$$
\frac{2}{q}+\frac{n}{r_{1}}<\frac{n}{2}
$$

hence, for any fixed value of $s$, the new Schrödinger admissible couple $\left(q, r_{1}\right)$ lies on a parallel line below the corresponding case $s=0$.

Strichartz estimates in Wiener amalgam spaces enable us to control the local regularity and decay at infinity of the solution separately. For comparison, the classical estimates (35) can be rephrased in terms of Wiener amalgam spaces as follows:

$$
\left\|e^{i t \Delta} u_{0}\right\|_{W\left(L^{q}, L^{q}\right)_{t} W\left(L^{r}, L^{r}\right)_{x}} \lesssim\left\|u_{0}\right\|_{L_{x}^{2}}
$$

In this framework, Cordero and Nicola perform these estimates mainly in two directions. First, in [6], for $q \geqslant 4$ they modify the classical estimate (40) by (conveniently) moving local regularity from the time variable to the space variable. Indeed, $\mathscr{F} L^{r^{\prime}} \subset L^{r}$ if $r \geqslant 2$, but the bound in (31) is worse than the one in (23), as $t \rightarrow 0$; consequently one has

$$
\left\|e^{i t \Delta} u_{0}\right\|_{W\left(L^{q / 2}, L^{q}\right)_{t} W\left(\mathscr{F} L^{r^{\prime}}, L^{r}\right)} \lesssim\left\|u_{0}\right\|_{L_{x}^{2}}
$$

for $4<q \leqslant \infty, 2 \leqslant r \leqslant \infty$, with $(q, r)$ Schrödinger admissible. When $q=4$ the same estimate holds with the Lorentz space $L^{r^{\prime}, 2}$ in place of $L^{r^{\prime}}$. Dual homogeneous and retarded estimates hold as well. Thereby, the solution averages locally in time by the $L^{q / 2}$ norm, which is rougher than the $L^{q}$ norm in (35) or, equivalently, in (40), but it displays an $\mathscr{F} L^{r^{\prime}}$ behavior locally in space, which is better than $L^{r}$. In [8] it is shown the sharpness of these Strichartz estimates, except for the threshold $q \geq 4$, which seems quite hard to obtain. Secondly, in [8], a converse approach is performed, by showing that it is possible to move local regularity in (35) from the space variable to the time variable. As a result, new estimates involving the Wiener amalgam spaces $W\left(L^{p}, L^{q}\right)$, that generalize (35), are obtained, i.e., the following [8, Theorem 1.1].

Theorem 4.5. Let $1 \leq q_{1}, r_{1} \leq \infty, 2 \leq q_{2}, r_{2} \leq \infty$ such that $r_{1} \leq r_{2}$,

$$
\begin{aligned}
& \frac{2}{q_{1}}+\frac{d}{r_{1}} \geq \frac{d}{2} \\
& \frac{2}{q_{2}}+\frac{d}{r_{2}} \leq \frac{d}{2}
\end{aligned}
$$


$\left(r_{1}, d\right) \neq(\infty, 2),\left(r_{2}, d\right) \neq(\infty, 2)$ and, if $d \geq 3, r_{1} \leq 2 d /(d-2)$. Assume the same for $\tilde{q}_{1}, \tilde{q}_{2}, \tilde{r}_{1}, \tilde{r}_{2}$. Then, we have the homogeneous Strichartz estimates

$$
\left\|e^{i t \Delta} u_{0}\right\|_{W\left(L^{q_{1}}, L^{q_{2}}\right)_{t} W\left(L^{r_{1}}, L^{r_{2}}\right)_{x}} \lesssim\left\|u_{0}\right\|_{L_{x}^{2}},
$$

the dual homogeneous Strichartz estimates

$$
\left\|\int e^{-i s \Delta} F(s) d s\right\|_{L^{2}} \lesssim\|F\|_{W\left(L^{\tilde{q}_{1}^{\prime}}, L^{\tilde{q}_{2}^{\prime}}\right)_{t} W\left(L^{\tilde{r}_{1}^{\prime}}, L^{\tilde{r}_{2}^{\prime}}\right)_{x},}
$$

and the retarded Strichartz estimates

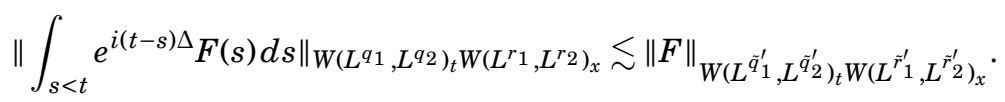

This outcome is achieved by first establishing the estimates for the particular case $q_{1}=$ $\tilde{q_{1}}=\infty, r_{1}=\tilde{r}_{1}=2$, and then by complex interpolation with the classical ones (35).

Figure 1 illustrates the range of exponents for the homogeneous estimates when $d \geq$ 3. Notice that, if $q_{1} \leq q_{2}$, these estimates follow immediately from (40) and the inclusion relations of Wiener amalgam spaces. So, the issue consists in the cases $q_{1}>q_{2}$. Since there are no relations between the pairs $\left(q_{1}, r_{1}\right)$ and $\left(q_{2}, r_{2}\right)$ other than $r_{1} \leq r_{2}$, these estimates tell us, in a sense, that the analysis of the local regularity of the Schrödinger propagator is quite independent of its decay at infinity.

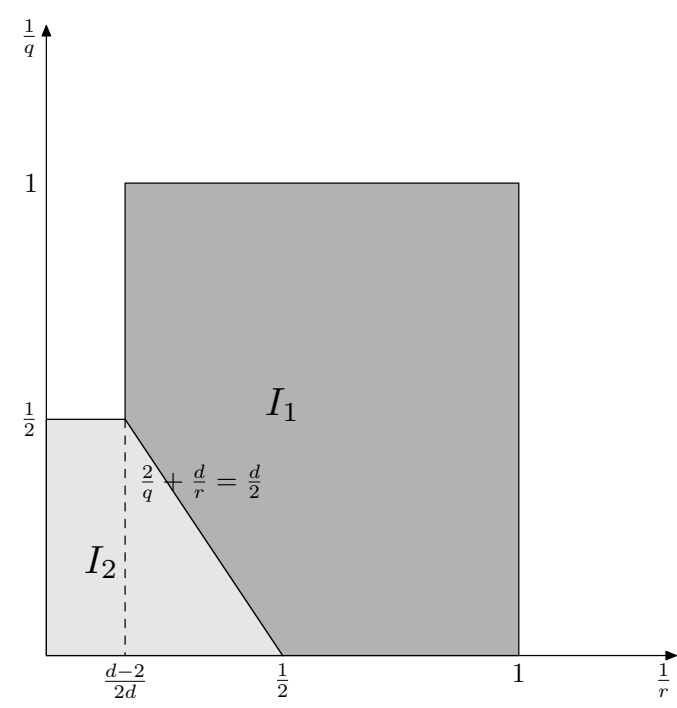

Figure 1: When $d \geq 3$, (44) holds for all pairs $\left(1 / q_{1}, 1 / r_{1}\right) \in I_{1},\left(1 / q_{2}, 1 / r_{2}\right) \in I_{2}$, with $1 / r_{2} \leq 1 / r_{1}$. 
In [8] it is proved that, for $d \geq 3$, all the constraints on the range of exponents in Theorem 4.5 are necessary, except for $r_{1} \leq r_{2}, r_{1} \leq 2 d /(d-2)$, which is still left open. However, the following weaker result holds [8, Proposition 5.3]:

Assume $r_{1}>r_{2}$ and $t \neq 0$. Then the propagator $e^{i t \Delta}$ does not map $W\left(L^{r_{1}^{\prime}}, L_{2}^{r_{2}^{\prime}}\right)$ continuously into $W\left(L^{r_{1}}, L^{r_{2}}\right)$.

shows that the estimates (44) for exponents $r_{1}>r_{2}$, if true, cannot be obtained from fixedtime estimates and orthogonality arguments. The arguments employed for the necessary conditions differ from the classical setting of Lebesgue spaces, because the general scaling consideration does not work directly. Indeed, the known bounds for the norm of the dilation operator $f(x) \longmapsto f(\lambda x)$ between Wiener amalgam spaces ([37, 40]), yield constraints which are weaker than the desired ones. So, the necessary conditions are obtained considering families of rescaled Gaussians as initial data, for which the action of the operator $e^{i t \Delta}$ and the involved norms can be computed explicitly, see [8].

We end up this section with recalling Stricharz estimates for modulation spaces. The main result in this framework is due to Wang and Hudzik [45]. They use the same arguments as in Keel and Tao [26], who point out that the ranges of exponents $(q, r)$ in (23) could most likely be not optimal. In fact, Keel and Tao show that if the semigroup $e^{i t \Delta}$ satisfies the estimate

$$
\left\|e^{i t \Delta} u_{0}\right\|_{L^{p}} \lesssim(1+|t|)^{-d(1 / 2-1 / p)}\left\|u_{0}\right\|_{L^{p^{\prime}}}
$$

then (35), (36) and (37) hold if one substitutes $q$ and $\tilde{q}$ by any $\gamma \geq \max (q, 2)$ and $\tilde{\gamma} \geqslant \max (\tilde{q}, 2)$, respectively. Since the estimate (34) is similar to (47), they optimize (35), (36) and (37) in the function spaces $M_{s}^{p, q}$ to cover the exponents $(\gamma, q)$ and $(\tilde{\gamma}, \tilde{q})$ satisfying $\gamma \geqslant \max (q, 2)$ and $\tilde{\gamma} \geqslant \max (\tilde{q}, 2)$. Since the precise formulation of these results requires the introduction of other function spaces, we refer interested readers to [45, Section 3].

\section{Applications}

We start by focusing on the Cauchy problem for the nonlinear Schrödinger equation (NLS)

$$
\left\{\begin{array}{l}
i \partial_{t} u+\Delta u+N(u)=0 \\
u(0, x)=u_{0}(x)
\end{array}\right.
$$

The nonlinearity $N$ considered will be either power-like

$$
p_{k}(u)=\lambda|u|^{2 k} u, \quad k \in \mathbb{N}, \lambda \in \mathbb{R}
$$

or exponential-like

$$
e_{\rho}(u)=\lambda\left(e^{\rho|u|^{2}}-1\right) u, \quad \lambda, \rho \in \mathbb{R} .
$$


Both nonlinearities are smooth. The corresponding equations having power-like nonlinearities $p_{k}$ are sometimes referred to as algebraic nonlinear Schrödinger equations. The sign of the coefficient $\lambda$ determines the defocusing, absent, or focusing character of the nonlinearity.

We shall study the well-posedness of (48), in different spaces. Recall that the problem (48) is locally well-posed in e.g. $H_{x}^{s}\left(\mathbb{R}^{d}\right)$ if, for any $u_{0}^{*} \in H_{x}^{s}\left(\mathbb{R}^{d}\right)$, there exists a time $T>0$ and an open ball $B$ in $H_{x}^{s}\left(\mathbb{R}^{d}\right)$ containing $u_{0}^{*}$ and a subset of $C_{t}^{0} H_{x}^{s}\left([T, T] \times \mathbb{R}^{d}\right)$ such that for each $u_{0} \in B$ there exists a unique solution $u \in X$ to the equation (48) and the map $u_{0} \mapsto u$ is continuous from $B$ (with the $H_{x}^{s}$ topology) to $X$ (with the $C_{t}^{0} H_{x}^{s}\left([T, T] \times \mathbb{R}^{d}\right.$ ) topology).

A fundamental tool in well-posedness theory is the contraction theorem. Let us first work abstractly, viewing (48) as an instance of the more general

$$
u=u_{\text {lin }}+D N(u)
$$

where $u_{\text {lin }}:=e^{i t \Delta} u_{0}$ is the linear solution, $N$ is the nonlinearity and $D$ is the Duhamel operator

$$
D F(t, x):=\int_{0}^{t} e^{i(t-s) \Delta} F(s, \cdot) d s .
$$

The following abstract tool [39, Proposition 1.38] then allows us to find the desired contraction map.

Proposition 5.1 (Abstract iteration argument). Let $\mathscr{N}, \mathscr{T}$ be two Banach spaces. Let $D$ : $\mathscr{N} \rightarrow \mathscr{T}$ be a bounded linear operator with the bound

$$
\|D F\|_{\mathscr{T}} \leqslant C_{0}\|F\|_{\mathscr{N}}
$$

for all $F \in \mathscr{N}$ and some constant $C_{0}>0$, and let $N: \mathscr{S} \rightarrow \mathscr{N}$, with $N(0)=0$, be a nonlinear operator which is Lipschitz continuous and obeys the bounds

$$
\|N(u)-N(v)\|_{\mathscr{N}} \leqslant \frac{1}{2 C_{0}}\|u-v\|_{\mathscr{T}}
$$

for all $u, v$ in the ball $B_{\epsilon}:=\left\{u \in \mathscr{S}:\|u\|_{\mathscr{T}} \leqslant \epsilon\right\}$, for some $\epsilon>0$. Then, for all $u_{\text {lin }} \in B_{\epsilon / 2}$, there exists a unique solution $u \in B_{\epsilon}$ to the equation (49), with Lipschitz map $u_{\text {lin }} \mapsto u$ with constant at most 2 . That is, we have

$$
\|u\|_{\mathscr{T}} \leqslant 2\left\|u_{\text {lin }}\right\|_{\mathscr{T}}
$$

Proof. Observe that for $v=0$ the estimate (51) becomes

$$
\|N(u)\|_{\mathscr{N}} \leqslant \frac{1}{2 C_{0}}\|u\|_{\mathscr{T}}
$$

( since $N(0)=0$ by hypothesis). Then, fix $u_{\operatorname{lin}} \in B_{\epsilon / 2}$, and consider the map

$$
\phi(u):=u_{\text {lin }}+D N(u) .
$$


Using (50) and (53) one has

$$
\|\phi(u)\|_{\mathscr{T}}=\left\|u_{\operatorname{lin}}+D N(u)\right\|_{\mathscr{T}} \leqslant \frac{\epsilon}{2}+\frac{C_{0}}{2 C_{0}} \epsilon=\epsilon
$$

for all $u \in B_{\epsilon}$, i.e., $\phi$ maps the ball $B_{\epsilon}$ into $B_{\epsilon}$. Moreover, $\phi$ is a contraction on $B_{\epsilon}$, indeed by (50) and (51) one has

$$
\begin{aligned}
\|\phi(u)-\phi(v)\|_{\mathscr{T}} & =\|D N(u)-D N(v)\|_{\mathscr{T}} \leqslant C_{0}\|N(u)-N(v)\|_{\mathscr{N}} \\
& \leqslant C_{0} \frac{1}{2 C_{0}}\|u-v\|_{\mathscr{T}}=\frac{1}{2}\|u-v\|_{\mathscr{T}},
\end{aligned}
$$

for all $u, v \in B_{\epsilon}$. Then, the contraction theorem asserts that there exists a unique fixed point $u$ for $\phi$ and moreover the map $u_{\operatorname{lin}} \mapsto u$ is Lipschitz with constant at most 2, that is (52).

Proposition 5.1 is the main ingredient of the results in [1, 7, 8, 9, 39, 45].

First, consider the NLS (48) with $N=p_{k}$, with the initial data $u_{0}$ in the Sobolev space $H_{x}^{s}\left(\mathbb{R}^{d}\right)$. To study this Cauchy problem it is convenient to introduce a single space $S^{s}$ that recaptures all the Strichartz norms at a certain regularity $H_{x}^{s}\left(\mathbb{R}^{d}\right)$ simultaneously. For sake of simplicity, we reduce to the case $s=0$ which corresponds to the case $L_{x}^{2}$, introducing the Strichartz space $S^{0}\left(I \times \mathbb{R}^{d}\right)$, for any time interval $I$, defined as the closure of Schwartz class $\mathscr{S}$ with respect to the norm

$$
\|u\|_{\mathscr{S}^{0}\left(I \times \mathbb{R}^{d}\right)}:=\sup _{A}\|u\|_{L_{t}^{q} L_{x}^{r}\left(I \times \mathbb{R}^{d}\right)},
$$

where the set $A$ is given by $A:=\{(\infty, 2),(q, r)\}$, with $(q, r)$ Schrödinger admissible. We define also the space $N^{0}\left(I \times \mathbb{R}^{d}\right):=L_{t}^{q^{\prime}} L_{x}^{r^{\prime}}$. Then, using Proposition 5.1 and the $L^{p}$ Strichartz estimates of Theorem 4.3 one can prove the following [39, Proposition 3.15]

Theorem 5.2 ( $L_{x}^{2}$ subcritical solution). Let $k$ be subcritical for $L_{x}^{2}$ (that is, $0<k<\frac{2}{d}$ ) and let $\mu= \pm 1$. Then the NLS (48) is locally well-posed in $L_{x}^{2}$ in a subcritical sense. Indeed, for any $R>0$ there exists a time $T>0$ such that for all $u_{0}$ in the ball $B_{R}:=\left\{u_{0} \in L_{x}^{2}\left(\mathbb{R}^{d}\right):\left\|u_{0}\right\|_{L_{x}^{2}}<R\right\}$ there exists a unique solution $u$ in $L_{x}^{2}$ of (48) in the space $\mathscr{S}^{0}\left([-T, T] \times \mathbb{R}^{d}\right) \subset C_{t}^{0} L_{x}^{2}\left([-T, T] \times \mathbb{R}^{d}\right)$. Moreover, the map $u_{0} \mapsto u$, from $B_{R}$ to $\mathscr{S}^{0}\left([-T, T] \times \mathbb{R}^{d}\right)$, is Lipschitz continuous.

For results in the framework of modulation spaces we address to [2, 44, 45]. In particular, we examine [2]. The main result, obtained only with the $M_{s}^{p, q}$ dispersive estimates (32), is the following.

Theorem 5.3. Assume that $u_{0} \in M_{s}^{p, 1}\left(\mathbb{R}^{d}\right)$ and $N \in\left\{p_{k}, e_{\rho}\right\}$. Then, there exists $T=T\left(\left\|u_{0}\right\|_{M_{s}^{p, 1}}\right)$ such that (48) has a unique solution $u \in C^{0} M_{s}^{p, 1}\left([0, T] \times \mathbb{R}^{d}\right)$. Moreover, if $T<\infty$, then $\lim \sup _{t \rightarrow T}\|u(t, \cdot)\|=\infty$. 
Proof. The proof is simply an application of the abstract iteration argument. Let us write it for the nonlinearity $N=p_{k}$. We choose the spaces $\mathscr{T}:=C^{0} M_{s}^{p, 1}([0, T] \times \mathbb{R}), \mathscr{N}:=M_{s}^{p, 1}$, and the Duhamel operator

$$
D:=\int_{0}^{t} e^{i(t-s) \Delta} \cdot d s .
$$

Then, it is sufficient to prove (50) and (51) in this setting. Then, by the Minkowsky integral inequality, $M_{s}^{p, q}$ dispersive estimates (32) and [2, Corollary 2] one has

$$
\begin{aligned}
\left\|\int_{0}^{t} e^{i(t-\tau) \Delta}\left(p_{k}(u)\right)(\tau) d \tau\right\|_{M_{s}^{p, 1}} & \leqslant \int_{0}^{t}\left\|e^{i(t-\tau) \Delta}\left(p_{k}(u)\right)(\tau)\right\|_{M_{s}^{p, 1}} d \tau \\
& \leqslant c_{1} T C_{T} \sup _{t \in[0, T]}\left\|p_{k}(u)(t)\right\|_{M_{s}^{p, 1}} \\
& \leqslant c_{1} c_{2} C_{T} T\|u(t)\|_{M_{s}^{p, 1}}^{2 k+1}
\end{aligned}
$$

where $C_{T}=\sup _{t \in[0, T)}(1+|t|)^{d / 2}$. Choosing $T>0$ such that $c_{1} c_{2} C_{T} T \leqslant C_{0}$, it follows (50) and by

$$
p_{k}(u)(\tau)-p_{k}(v)(\tau)=\lambda(u-v)|u|^{2 k}(\tau)+\lambda v\left(|u|^{2 k}-|v|^{2 k}\right)(\tau)
$$

it follows (51).

For Wiener amalgam spaces there are no results for the NLS. In [8] there is a result concerning linear Schrödinger equations with time-dependent potentials. Indeed, in [8] the well-posedness is proved in $L^{2}$ of the following Cauchy problem, for all $d \geq 1$,

$$
\left\{\begin{array}{l}
i \partial_{t} u+\Delta u=V(t, x) u, \quad t \in[0, T]=I_{T}, x \in \mathbb{R}^{d} \\
u(0, x)=u_{0}(x)
\end{array}\right.
$$

and for the class of potentials

$$
V \in L^{\alpha}\left(I_{T} ; W\left(\mathscr{F} L^{p^{\prime}}, L^{p}\right)_{x}\right), \quad \frac{1}{\alpha}+\frac{d}{p} \leq 1,1 \leq \alpha<\infty . d<p \leq \infty .
$$

Theorem 5.4. Consider the class of potentials (55). Then, for all $(q, r)$ such that $2 / q+d / r=d / 2$, $q>4, r \geq 2$, the Cauchy problem (54) has a unique solution

$$
\begin{aligned}
& \text { (i) } u \in \mathscr{C}\left(I_{T} ; L^{2}(\mathbb{R})\right) \cap L^{q / 2}\left(I_{T} ; W\left(\mathscr{F} L^{r^{\prime}}, L^{r}\right)\right) \text {, if } d=1 ; \\
& \text { (ii) } u \in \mathscr{C}\left(I_{T} ; L^{2}\left(\mathbb{R}^{d}\right)\right) \cap L^{q / 2}\left(I_{T} ; W\left(\mathscr{F} L^{r^{\prime}}, L^{r}\right)\right) \cap \\
& \cap L^{2}\left(I_{T} ; W\left(\mathscr{F} L^{2 d /(d+1), 2}, L^{2 d /(d-1)}\right)\right), \text { if } d>1 .
\end{aligned}
$$

Proof. It is enough to prove the case $d=1$. Indeed, for $d \geq 2$, condition (55) implies $p>2$, so that $\mathscr{F} L^{p^{\prime}} \hookrightarrow L^{p}$ and the inclusion relations of Wiener amalgam spaces yield $W\left(\mathscr{F} L^{p^{\prime}}, L^{p}\right) \hookrightarrow$ 
$W\left(L^{p}, L^{p}\right)=L^{p}$. Hence our class of potentials is a subclass of those of [6, Theorem 6.1], for which the quoted theorem provides the desired result.

We now turn to the case $d=1$. The proof follows the ones of [9, Theorem 1.1, Remark 1.3] and [6, Theorem 6.1] (see also [49]).

First of all, since the interval $I_{T}$ is bounded, by Hölder's inequality and by taking $p$ large, we may assume $1 / \alpha+d / p=1$.

We choose a small time interval $J=[0, \delta]$ and set, for $q \geq 2, q \neq 4, r \geq 1$,

$$
Z_{q / 2, r}=L^{q / 2}\left(J ; W\left(\mathscr{F} L^{r^{\prime}}, L^{r}\right)_{x}\right) .
$$

Now, fix an admissible pair $\left(q_{0}, r_{0}\right)$ with $r_{0}$ arbitrarily large (hence $\left(1 / q_{0}, 1 / r_{0}\right)$ is arbitrarily close to $(1 / 4,0))$ and set $Z=\mathscr{C}\left(J ; L^{2}\right) \cap Z_{q_{0} / 2, r_{0}}$, with the norm $\|v\|_{Z}=\max \left\{\|v\|_{\mathscr{C}\left(J ; L^{2}\right)},\|v\|_{Z_{q_{0} / 2, r_{0}}}\right\}$. We have $Z \subset Z_{q / 2, r}$ for all admissible pairs $(q, r)$ obtained by interpolation between $(\infty, 2)$ and $\left(q_{0}, r_{0}\right)$. Hence, by the arbitrary of $\left(q_{0}, r_{0}\right)$ it suffices to prove that $\Phi$ defines a contraction in $Z$.

Consider now the integral formulation of the Cauchy problem, namely $u=\Phi(v)$, where

$$
\Phi(v)=e^{i t \Delta} u_{0}+\int_{0}^{t} e^{i(t-s) \Delta} V(s) v(s) d s .
$$

By the homogeneous and retarded Strichartz estimates in [6, Theorems 1.1, 1.2] the following inequalities hold:

$$
\|\Phi(v)\|_{Z_{q / 2, r}} \leq C_{0}\left\|u_{0}\right\|_{L^{2}}+C_{0}\|V v\|_{Z_{(\tilde{q} / 2)^{\prime}, \tilde{r}^{\prime}}}
$$

for all admissible pairs $(q, r)$ and $(\tilde{q}, \tilde{r}), q>4, \tilde{q}>4$.

Consider now the case $1 \leq \alpha<2$. We choose $\left((\tilde{q} / 2)^{\prime}, \tilde{r}\right)=(\alpha, 2 p /(p+2))$. Since $v \in L^{\infty}\left(J ; L^{2}\right)$, applying (13) for $q=2$ we get

$$
\|V v\|_{W\left(\mathscr{F} L^{\tilde{r}}, L^{\tilde{r}^{\prime}}\right)} \lesssim\|V\|_{W\left(\mathscr{F} L^{p^{\prime}}, L^{p}\right)}\|v\|_{L^{2}}
$$

whereas Hölder's Inequality in the time-variable gives

$$
\|V v\|_{(\tilde{q} / 2)^{\prime}, \tilde{r}^{\prime}} \lesssim\|V\|_{L^{\alpha}\left(J ; W\left(\mathscr{F} L^{p^{\prime}}, L^{p}\right)\right)}\|v\|_{L^{\infty}\left(J ; L^{2}\right)} .
$$

The estimate (56) then becomes

$$
\|\Phi(v)\|_{Z_{q / 2, r}} \leq C_{0}\left\|u_{0}\right\|_{L^{2}}+C_{0}\|V\|_{L^{\alpha}\left(J ; W\left(\mathscr{F} L^{p^{\prime}}, L^{p}\right)\right)}\|v\|_{L^{\infty}\left(J ; L^{2}\right)} .
$$

By taking $(q, r)=(\infty, 2)$ or $(q, r)=\left(q_{0}, r_{0}\right)$ one deduces that $\Phi: Z \rightarrow Z$ (the fact that $\Phi(u)$ is continuous in $t$ when valued in $L_{x}^{2}$ follows from a classical limiting argument [9, Theorem 1.1, Remark 1.3]). Also, if $J$ is small enough, $C_{0}\|V\|_{L_{t}^{\alpha} L_{x}^{p}}<1 / 2$, and $\Phi$ is a contraction. This gives 
a unique solution in $J$. By iterating this argument a finite number of times one obtains a solution in $[0, T]$.

The case $2 \leq \alpha<\infty$ is similar.

This result generalizes [6, Theorem 6.1], by treating the one dimensional case as well and allowing the potentials to belong to Wiener amalgam spaces with respect to the space variable $x$. Other results on Schrödinger equations with potentials in $L_{t}^{p} L_{x}^{q}$ can be found in [9].

\section{References}

[1] Bényi, A., Gröchenig, K., Okoudjou, K.A. And Rogers, L.G., Unimodular Fourier multipliers for modulation spaces, J. Funct. Anal., 246(2), 366-384, 2007.

[2] BÉNyI, A. AND OKoudJou, K.A., Local well-posedness of nonlinear dispersive equations on modulation spaces, Bulletin of the London Mathematical Society, 2009.

[3] Berezin, F.A. AND Shubin, M.A., The Schrödinger equation, Mathematics and its Applications (Soviet Series), 66, Kluwer Academic Publishers Group, 1991.

[4] Bergh, J. AND LÖFström, J., Interpolation Spaces. An Introduction, Springer-Verlag, Berlin, 1976. Grundlehren der Mathematischen Wissenschaften, No. 223.

[5] Cazenave, T. and Weissler, F.B., The Cauchy problem for the critical nonlinear Scrödinger equation in $H^{s}$, Nonlinear Anal., 14, 807-836, 1990.

[6] Cordero, E. AND Nicola, F., Strichartz estimates in Wiener amalgam spaces for the Schrödinger equation, Math. Nachr., 281(1), 25-41, 2008.

[7] Cordero, E. ANd Nicola, F., Metaplectic representation on Wiener amalgam spaces and applications to the Schrödinger equation, J. Funct. Anal., 254(2), 506-534, 2008.

[8] Cordero, E. ANd Nicola, F., Some new Strichartz estimates for the Schrödinger equation, J. Differential Equation, 245, 1945-1974, 2008.

[9] D'Ancona, P., Pierfelice, V. And Visciglia, N., Some remarks on the Schrödinger equation with a potential in $L_{t}^{r} L_{x}^{s}$, Math. Ann., 333(2), 271-290, 2005.

[10] D’Ancona, P., Non Linear Waves, seminary notes, Cortona, Luglio 2007, 205(1):107$131,2003$.

[11] DE Gosson, M., The quantum motion of half-densities and the derivation of Schrödinger's equation, J. Phys. A: Math. Gen., 31, 4239-4247, 1998. 
[12] Feichtinger, H.G., Banach convolution algebras of Wiener's type, In Proc. Conf. "Function, Series, Operators", Budapest August 1980, Colloq. Math. Soc. János Bolyai, 35, 509-524, North-Holland, Amsterdam, 1983.

[13] Feichtinger, H.G., Modulation spaces on locally Abelian groups, Technical Report, University of Vienna, 1983, updated version appeared in Proceedings of International Conference on Wavelets and Applications, Chennai, India, 2003, pp. 99-140.

[14] Feichtinger, H.G., Banach spaces of distributions of Wiener's type and interpolation, In Proc. Conf. Functional Analysis and Approximation, Oberwolfach August 1980, Internat. Ser. Numer. Math., 69, 153-165. Birkhäuser, Boston, 1981.

[15] Feichtinger, H.G., Generalized amalgams, with applications to Fourier transform, Canad. J. Math., 42(3), 395-409, 1990.

[16] Fournier, J.J.F. And Stewart, J., Amalgams of $L^{p}$ and $l^{q}$, Bull. Amer. Math. Soc. (N.S.), 13(1), 1-21, 1985.

[17] Feichtinger, H.G. And Zimmermann, G., A Banach space of test functions for Gabor analysis, In Feichtinger, H.G. and Strohmer, T., editors, Gabor Analysis and Algorithms. Theory and Applications, Applied and Numerical Harmonic Analysis, 123-170, Birkhäuser, Boston, 1998.

[18] Foschi, D., Inhomogeneous Strichartz estimates, Jour. Hyperbolic Diff. Eqs., 2(1), 1-24, 2005.

[19] Ginibre, J. AND Velo, G., Smoothing properties and retarded estimates for some dispersive evolution equations, Comm. Math. Phys., 144(1), 163-188, 1992.

[20] Ginibre, J. AND VELO, G., Generalized Strichartz Inequalities for the Wave Equation, J. Funct. Anal., 133, 50-68, 1995.

[21] Gröchenig, K., Foundation of Time-Frequency Analysis, Birkhäuser, Boston MA, 2001.

[22] Heil, C., An introduction to weighted Wiener amalgams, In Krishna, M., Radha, R. and Thangavelu, S., editors, Wavelets and their Applications, 183-216, Allied Publishers Private Limited, 2003.

[23] Hörmander, L., Estimates for translation invariant operators in $L^{p}$ spaces, Acta Math., 104, 93-140, 1960.

[24] Kato, T., Linear evolution equations of "hyperbolic" type, J. Fac. Sci. Univ. Tokyo Sect. I, 17, 241-258, 1970. 
[25] Kato, T., An $L^{q, r}$-theory for nonlinear Schrödinger equations, Spectral and scattering theory and applications, Adv. Stud. Pure Math. Math. Soc. Japan, Tokyo, 23, 223-238, 1994.

[26] Keel, M. And TaO, T., Endpoint Strichartz estimates, Amer. J. Math., 120, 955-980, 1998.

[27] Machinara, S., Nakamura, M., Nakanishi, K. and Ozawa, T., Endpoint Strichartz estimates and global solutions for the nonlinear Dirac equation, J. Func. Anal., 219, 1-20 2005.

[28] Montgomery-Smith, S.J., Time decay for the bounded mean oscillation of solutions of the Schrödinger and wave equation, Duke Math. J., 19, 393-408, 1998.

[29] Nicola, F., Remarks on dispersive estimates and curvature, Commun. Pure Appl. Anal., 6, 203-212, 2007.

[30] Oberlin, D.M., Convolution estimates for some distributions with singularities on the light cone, Duke Math. J., 59(3), 747-757, 1989.

[31] SEgAL, I.E., Space-time decay for solutions of wave equations, Adv. Math., 22, 304-311, 1976.

[32] Stefanov, A., Strichartz estimates for the Schrödinger equation with radial data, Proc. Amer. Math. Soc., 129(5), 1395-1401, 2001.

[33] Stein, E.M., Singular integrals and differentiability properties of functions, Princeton University Press, Priceton, 1970.

[34] Stein, E.M., Harmonic analysis, Princeton University Press, Priceton, 1993.

[35] Stein, E.M. And Weiss, G., Introduction to Fourier Analysis on Euclidean spaces, Princeton University Press, 1971.

[36] STRICHARTZ, R.S., Restriction of Fourier transform to quadratic surfaces and decay of solutions of wave equations, Duke Math. J., 44, 705-774, 1977.

[37] Sugimoto, M. And Tomita, N., The dilation property of modulation spaces and their inclusion relation with Besov spaces, J. Funct. Anal., 248, n.1, 79-106, 2009.

[38] TAO, T., Spherically averaged endpoint Strichartz estimates for the two-dimensional Schrödinger equation, Comm. Partial differential Equations, 25, 1471-1485, 2000.

[39] TAO, T., Nonlinear Dispersive Equations: Local and Global Analysis, CBMS Regional Conference Series in Mathematics, Amer. Math. Soc., 2006. 
[40] TofT, J., Continuity properties for modulation spaces, with applications to pseudodifferential calculus. I, J. Funct. Anal., 207(2), 399-429, 2004.

[41] Tomas, P., A restriction theorem for the Fourier transform, Bull. Amer. Math. Soc., 81(1), 477-478, 1975.

[42] TRIEBEL, H., Interpolation theory, function spaces, differential operators, NorthHolland, 1978.

[43] VIlela, M.C., Strichartz estimates for the nonhomogeneous Schrödinger equation, Trans. Amer. Math. Soc., 359(5), 2123-2136, 2007.

[44] WANG, B., ZHAO, L. AND Guo, B., Isometric decomposition operators, function spaces $E_{p, q}^{\lambda}$ and applications to nonlinear evolution equations, J. Funct. Anal., 233(1), 1-39, 2006.

[45] Wang, B. AND HUdzIK, H., The global Cauchy problem for the NLS and NLKG with small rough data, J. Differential Equations, 232, 36-73, 2007.

[46] Wiener, N., On the representation of functions by trigonometric integrals, Math. Z., 24, 575-616, 1926.

[47] Wiener, N., Tauberian theorems, Ann. of Math., 33, 1-100, 1932.

[48] Wiener, N., The Fourier integral and certain of its application, MIT Press Cambridge, 1933.

[49] Yajima, K., Existence of solutions for Schrödinger evolution equations, Comm. Math. Phys., 110(3), 415-426, 1987.

[50] Zucco, D., Stime dispersive e di Strichartz per l'equazione di Schrödinger, Thesis, University of Torino, 2008. 